\title{
XXXV. Notiz über die Zusammensetzung des Ilmenits.
}

Von

S. I. Penfield und H. W. Foote in New Haven, Conn.

Die Existenz eines Moleküls $\stackrel{\text { II }}{R O}$. $\stackrel{\text { IV }}{O_{2}}$ im Bixbyit und Perowskit erinnert an die verschiedenen Ansichten über die Zusammensetzung des Ilmenits. Nach der einen derselben kommt dem Mineral die Formel $\mathrm{RO} \mathrm{TiO}_{2}(\boldsymbol{R}=\mathrm{Fe}$ und $M g$ ) zu, welche zuerst von Mosander aufgestellt (Pogg. Ann. 19, 219) und von Rammelsberg (Pogg. Ann. 104, 497) und Hamberg (diese Zeitschrift 1892, 20,395) angenommen wurde. Nach der anderen ist die $\mathrm{Zu}-$ sammensetzung $\mathrm{R}_{2} \mathrm{O}_{3}$ oder eine isomorphe Mischung von $\mathrm{Fe}_{2} \mathrm{O}_{3}$ und $\mathrm{Ti}_{2} \mathrm{O}_{3}$, wie Rose vorschlägt (Pogg. Ann. 62, 119) und Groth annimmt (Tabell. Uebersicht d. Min. 3. Aufl. 1889, 40).

Sowohl krystallographische als chemische Grunde zur Annahme von Mosander's Formel liegen vor. Hämatit und künstliches $\mathrm{Ti}_{2} \mathrm{O}_{3}$ krystallisiren beide in der rhomboëdrisch-hemiëdrischen Klasse des hexagonalen Systems, und die Länge ihrer Verticalaxe ist 1,359 resp. 1,316. Ilmenit jedoch unterscheidet sich in seiner Symmetrie von den vorbergehenden, indem er in der rhomboëdrisch-tetartoëdrischen Klasse des hexagonalen Systems krystallisirt, und die Länge seiner Verticalaxe, 1,385 , nicht zwischen der des Hämatits und Titansesquioxyds liegt, wie man erwarten sollte, wenn Ilmenit eine isomorphe Mischung von $\mathrm{Fe}_{2} \mathrm{O}_{3}$ und $\mathrm{Ti}_{2} \mathrm{O}_{3}$ wäre. Ueberdies möchte man beim Isomorphismus der beiden Sesquioxyde erwarten, dass einmal auch $\mathrm{Ti}_{2} \mathrm{O}_{3}$ im Ueberschusse vorhanden sein möchte, was noch niemals beobachtet wurde, obgleich das Verhältniss von $F e: T i$ in einigen Fällen fast genau $1: 1$ betrug.

Die Gegenwart von Magnesia in fast allen untersuchten Ilmeniten kann bei der Annabme, dass das Mineral eine isomorphe Mischung der Sesquioxyde $\mathrm{Fe}_{2} \mathrm{O}_{3}$ und $\mathrm{Ti}_{2} \mathrm{O}_{3}$ sei, nicht erklärt werden.

Die Menge der Magnesia ist gewöhnlich gering, weniger als $\mathbf{5} \%$, aber Goben (N. Jahrb. f. Min. 1877, 695) hat einen Ilmenit von Du Toit's Pan, Sudafrika, beschrieben, welcher in gerundeten Körnern vorkommt und $12,10 \% M g O$ enthält und Rammelsberg (l. c.) einen krystallisirten von 
Layton's Farm, Warwick, N. Y., welcher $13,71 \% \mathrm{MgO}$ enthielt. Groth bemerkt zur Analyse Rammelsberg's, dass das Material unrein gewesen sein möchte, und wir hielten es, da uns in der Brush'schen Sammlung einige vorzuglich krystallisirte Stücke von diesem Fundorte zu Gebote standen, fur das beste, eine neve Analyse zu machen. Das Material stammte von einem einzelnen Krystalle. Dieser war uneben, so dass keine genauen Messungen angestellt werden konnten, aber der Habitus war der des Ilmenits und mittelst des Contactgoniometers liessen sich die Formen $c\{0001\}, r\{10 T 1\}$ und $s\{02 \overline{2} 1\}$ identificiren. Die Analyse (von Foote) ist unten zusammen mit der von Rammelsberg ausgefuhrten angegeben.

\begin{tabular}{|c|c|c|c|c|c|c|}
\hline & 1. & II. & Mittel: & Verhâltniss: & Rammelsb.: & Verhälıniss : \\
\hline $\mathrm{SiO}_{2}$ & 0,44 & 0,31 & 0,37 & $0,006)$ & - & - \\
\hline $\mathrm{TiO}_{2}$ & 57,30 & 57,28 & 57,29 & $0,716\}^{0,72,2}$ & 57,71 & 0,721 \\
\hline $\mathrm{HeO}$ & 24,08 & 24,23 & 24,15 & 0,335 & 26,82 & 0,372 \\
\hline $\mathrm{MgO}$ & 16,01 & 15,93 & 15,97 & $0,399\} 0,749$ & 13,71 & $0,342\} 0,727$ \\
\hline $\mathrm{MnO}$ & 1,09 & 1,12 & 1,10 & 0,015 & 0,90 & 0,013 \\
\hline $\mathrm{Fe}_{2} \mathrm{O}_{3}$ & 1,99 & 1,75 & 1,87 & 0,012 & 一 & \\
\hline & 100,91 & 100,62 & 100,75 & & 99,14 & \\
\hline Spec. & Jewicht & & 4,345 & & 4,303 & \\
\hline
\end{tabular}

In beiden Analysen ist das Verbältniss von $R_{2} O: R O$ sehr nahe 1: 1, was die Existenz des Molekuls $\mathrm{RO}^{\mathrm{TiO}} \mathrm{O}_{2}$, wo $R=\mathrm{Fe}$ und $\mathrm{Mg}$ ist, nachweist.

Es hat sich also endgiltig erwiesen, dass in dieser krystallisirten Variëtăt des Ilmenits ein Molekul $\mathrm{MgO}^{\mathrm{TiO}} \mathrm{O}_{2}$ oder $\mathrm{MgTiO}_{3}$ vorliegt, und es scheint höchst einleuchtend, vorauszusetzen, dass das Eisen auch als $\mathrm{FeO}$. $\mathrm{TiO}_{2}$ vorhanden ist, isomorph mit $\mathrm{MgO}^{\mathrm{TiO}} \mathrm{O}_{2}$, und nicht als isomorphe Mischung von $\mathrm{Fe}_{2} \mathrm{O}_{3}$ und $\mathrm{Ti}_{2} \mathrm{O}_{3}$. Es kann jedoch durch chemische Mittel nicht festgestellt werden, dass das gesammle Titan vierwerthig vorliegt, weil beim Auflösen des Minerals zur Analyse $\mathrm{Ti}_{2} \mathrm{O}_{3}$, wenn es zugegen ist, zu $\mathrm{TiO}_{2}$ oxydirt wird auf Kosten des $\mathrm{Fe}_{9} \mathrm{O}_{3}$, und die Analyse ein Aequivalent $\mathrm{FeO}$ ergeben muss $\left(\mathrm{Fe}_{2} \mathrm{O}_{3}+\mathrm{Ti}_{2} \mathrm{O}_{3}=2 \mathrm{TiO}_{2}+2 \mathrm{FeO}\right)$.

In den veröffentlichten Analysen des llmenits, wo das Verhältniss $\mathrm{TiO}_{2}$ : $R O$ sehr constant 1:1 ist, ist fast ausnahmslos ein Ueberschuss an $\mathrm{Fe}_{2} \mathrm{O}_{3}$ vorhanden, der in einigen Fällen mebrere Procent beträgt, wie man aus dem Verzeichniss der Analysen in Dana's System der Mineralogie S. 218 ersehen kann, und wie Hamberg betont hat, ist die Annahme zulässig, dass das Hämatitmolekul $\mathrm{Fe}_{2} \mathrm{O}_{3}$ oder $\mathrm{FeFeO}_{3}$ befähigt sei, sich mit dem Ilmenitmolekül $\mathrm{FeTiO}_{3}$ und $\mathrm{MgTiO}_{3}$ zu mischen, gerade wie $\mathrm{CaCO}_{3}$ und $\mathrm{NaCO}_{3}$ praktisch als isomorph betrachtet werden können. 